\title{
Investigating the Potential of FGMs through Numerical Minimization of Thermal Stresses
}

\author{
S. Van den Kerkhof ${ }^{a}$, M. Blommaert ${ }^{a}$, J. W. Coenen ${ }^{b}$, S. \\ Heuer $^{b}$, M. Baelmans ${ }^{a}$ \\ ${ }^{a}$ KU Leuven, Department of Mechanical Engineering, 3001 Leuven, Belgium \\ ${ }^{b}$ Forschungszentrum Jülich GmbH, Institut für Energie- und Klimaforschung - \\ Plasmaphysik, Partner of the Trilateral Euregio Cluster (TEC), 52425 Jülich, \\ Germany \\ E-mail: sander.vandenkerkhof@kuleuven.be
}

August 2017

\begin{abstract}
Functionally graded materials (FGMs) have the potential to reduce high stress concentrations near material interfaces.In the ITER divertor monoblock, this may be achieved by replacing the distinct $\mathrm{W}-\mathrm{Cu}$ interface by an FGM, which gradually changes the coefficient of thermal expansion (CTE) from copper to tungsten. To assess the full potential of FGMs in improving the thermal and mechanical behavior, a first proof-of-principle study is performed in this paper to numerically optimize the tungsten volume fraction distribution of a W-Cu FGM based ITER monoblock for minimal thermal stresses using a simple thermal conduction model. We show that the optimizer can ensure that the temperature remains below the recrystallization temperature of tungsten and that it minimizes the chosen cost function, based on the fractional margin between the local temperature and melting temperature, in a computationally efficient way. Furthermore, the resulting design has a lower Von Mises stress measure at the original position of the $\mathrm{W}-\mathrm{Cu}$ interface. However, a new material interface is introduced, which results in an additional undesired stress concentration. Future work should therefore aim at integrating a complete stress evaluation in the optimization method.
\end{abstract}

Keywords: numerical optimization, functionally graded materials, adjoint gradient approach, thermal stress simulation/minimization 


\section{Introduction}

One of the major challenges on the road towards economically viable fusion energy is coping with the large peak heat load impinging on the divertor target, and the corresponding elevated material temperature profiles and thermal stresses [1-5]. For ITER, the divertor target will be composed of monoblocks, which are beam-shaped tungsten blocks fixed on a $\mathrm{CuCrZr}$ cooling tube with a $\mathrm{Cu}$ interlayer $[2,6]$. However, this design has several disadvantages. First, stress concentrations are likely to establish near the material interfaces, since the CTE of tungsten is roughly four times smaller than that of copper. Second, due to the rather low thermal conductivity of tungsten, elevated temperatures are found at the top of the monoblock, which approach the recrystallization temperature, $T_{\text {rec }} \approx 1200{ }^{\circ} \mathrm{C}$. At this temperature, the mechanical properties of tungsten might severely deteriorate. Finally, at the bottom of the monoblock, the temperature might be below $200^{\circ} \mathrm{C}$ at which the ductile-to-brittle transition temperature (DBTT) of tungsten is reached. All these phenomena lead to accelerated crack growth and hence a decrease in divertor physical lifetime.

To overcome the issue of CTE mismatch, W$\mathrm{Cu}$ functionally graded materials (FGMs) are being studied as an option to avoid the macroscopic material interface by smoothly varying the volume fraction of $\mathrm{W}$ in the monoblock $[7,8]$. The following question then arises: what is the best possible distribution of the tungsten volume fraction to minimize the stresses and hence increase divertor lifetime? This question can be answered by making use of numerical optimization techniques, which have already been applied successfully to optimize the target shape and magnetic configuration to reduce the peak heat load [9-12].

The aim of this paper is twofold: first, showing the potential of FGMs to reduce the maximal temperature in the monoblock, while simultaneously decreasing the stress concentrations at the original W-Cu interface. Secondly, we demonstrate how powerful the adjointbased optimization strategy is as a design tool. In the remainder of this paper, we will first elaborate the model for the monoblock in section 2 and afterwards explain the optimization rationale in section 3 . In section 4 , the optimization strategy is used to optimize the tungsten volume fraction in the domain for a desired local temperature to melt temperature ratio, while constraining the maximum temperature to the recrystallization temperature of tungsten. Finally, the temperature distribution and Von Mises stress distribution in the optimized design are compared to those of the ITER reference case.

\section{A suitable divertor target model including FGMs}

The thermal behavior of the monoblock is modeled by the steady state heat conduction equation and adequate boundary conditions, denoted respectively by $c_{\Omega}$ and $c_{\Sigma}$ :

$c_{\Omega}\left(w_{W}, T\right)=\nabla \cdot\left(\kappa\left(w_{W}, T\right) \nabla T\right)=0$,

$c_{\Sigma}\left(w_{W}, T\right)=0$.

It is assumed here that the heat conduction is isotropic and no volumetric heat sources (e.g. heat originating from impinging neutrons) are present. The dependence of the model equations and heat conduction coefficient, $\kappa$, on the tungsten volume fraction, $w_{W}$, and on the state variables, in this case only the temperature $T$, expressed in $[\mathrm{K}]$, is explicitly stated. The following boundary conditions are imposed: at the plasma facing surface, a constant heat load of $10 \frac{\mathrm{MW}}{\mathrm{m}^{2}}$ is applied. The sides of the monoblock are assumed to be adiabatic and the cooling duct heat transfer is modeled by a correlation for subcooled boiling convective heat transport by water at $100^{\circ} \mathrm{C}$. These equations are solved using an in-house Finite Volume code for the $2 \mathrm{D}$ temperature distribution in the monoblock.

The heat conduction coefficient is taken from experimental data for a melt-injected W-Cu FGM from [13] to have a realistic representation of the expected FGM thermal behavior. Due to lack of available data, other material parameters, such as the Young's modulus, Poisson ratio, melting temperature, and CTE, are assumed to be given by a linear mixture model:

$\alpha_{F G M}=w_{W} \alpha_{W}+\left(1-w_{W}\right) \alpha_{C u}$,

where $\alpha_{W}$ and $\alpha_{C u}$ are the material parameters of pure tungsten or copper, respectively, and $w_{W}$ is the tungsten volume fraction.

\section{Introducing an efficient optimization methodology}

\subsection{Formulation of the optimization problem}

Ideally, we would like to adjust the tungsten volume fraction in the domain such that local stress concentrations are effectively reduced to a minimum, while at the same time keeping the temperature above the DBTT and below the recrystallization temperature of tungsten. This problem statement can be cast into an optimization problem where a cost function, $\mathcal{J}(\phi, T)$, is minimized by searching for the optimal values of the design variable $\phi$. The cost function is an indication for the performance of the design and should therefore correspond to some measure of the stresses, for example the Von Mises stress distribution in the 
monoblock. However, this requires solving the 3D stress distribution in the monoblock and may become computationally expensive. Therefore, another cost function is proposed which is cheap to evaluate, but still leads to local stress concentration reduction. Here, we opt to optimize the local temperature to melting temperature ratio, $\frac{T}{T_{m}}$, where $T_{m}$ is calculated using the linear mixture rule, and both temperatures are expressed in $[\mathrm{K}]$. In the remainder of this paper, this is also referred to as the temperature ratio. Since thermal stresses are at first instance proportional to the difference between local and stress free temperature, it is reasoned that this should lead to local stress reduction. Induced stresses related to temperature and CTE gradients are not directly taken into account, but it is reasoned that by reducing the overall relative temperature, the temperature profile and as such the stresses are smoothed out as well.

The design variable will be the spatial distribution of the tungsten volume fraction in the functionally graded material, i.e. $\phi=w_{W}$, on the design domain, $\Omega_{\phi}$, which is a part of the monoblock domain, $\Omega$. After discretizing the domain, the tungsten fraction in each cell of $\Omega_{\phi}$ becomes a design variable. This approach allows for maximal design freedom, yet might result in designs which are not easy realizable with current manufacturing techniques.

Besides minimizing the cost function, it is also possible to impose constraints. In our case, the first obvious constraint is that the design variables and temperature should satisfy the model equations, $c(\phi, T)=0$, under which we understand both the heat conduction equation $c_{\Omega}=0$ and the boundary conditions $c_{\Sigma}=0$. Further, the tungsten volume fraction should be positive and bounded by one, and the temperature should remain below the recrystallization temperature of tungsten. To reduce complexity, the DBTT constraint and the actual upper temperature limit of copper are not yet imposed. Note that the model equations can be solved and substituted directly for each choice of design parameters (tungsten distribution), leading to the reduced cost function, $\hat{\mathcal{J}}(\phi)$. Written succinctly, this gives the following optimization problem:

$$
\begin{array}{ll}
\min _{\phi} & \hat{\mathcal{J}}(\phi)=\frac{1}{2} \lambda \int_{\Omega}\left(\frac{T(\phi)}{T_{m}(\phi)}-\tau\right)^{2} d \omega . \\
\text { s.t. } & 0 \leq \phi \leq 1, \\
& T(\phi) \leq T_{\text {rec }} \quad \text { on } \quad \Omega .
\end{array}
$$

To stress that the model equations have already been solved, the dependence of the temperature on the design parameters is explicitly stated. For the cost function, it was chosen to optimize the L2-norm of $\frac{T}{T_{m}}-\tau$, normalized with a constant prefactor $\lambda$, since it is well known that the L2-norm leads to penalizing large peaks in the cost function value and will hence lead more quickly to local temperature ratio and hence stress reduction. $\tau$ represents the desired temperature ratio and should be chosen beforehand. This choice will be further clarified in section 4 .

\subsection{The adjoint approach: a cost-efficient way for gradient calculation}

To solve the aforementioned optimization problem efficiently, a gradient-based optimization algorithm will be used. To this end, the gradient of the reduced cost function and of the constraints are required to update the design, i.e. the tungsten fraction in each cell. An intuitive way to do this is by making use of finitedifference approximation to evaluate the gradient. However, with this approach, one has to perturb a design variable, recalculate the cost function (or constraint) for the perturbed design, and compare this to the original cost function. This should be repeated for every design variable. Therefore, this approach is computationally expensive: for each cost function calculation, the model equations should be solved. This in particular becomes prohibitively expensive for a large number of design variables, which in our case is in the order of $10^{4}-10^{5}$.

It is clear that an alternative strategy for gradient calculation is needed. The adjoint approach provides a solution: it allows to calculate the gradient directly for all design variables through the solution of the so-called adjoint equations, which are a set of equations similar to and derived from the (linearized) model equations, and the cost and constraint functions. This renders the cost of the gradient calculation independent of the number of design variables. Instead, it now scales with the number of cost and state-dependent constraint functions to be evaluated. The reader is referred to [9] for a detailed explanation and derivation.

\subsection{Dealing with the temperature constraint}

As briefly mentioned before, the gradient of the constraint with respect to the design variables is required as well. This is trivial for the bound constraints on the design variable, but hard for the maximal temperature constraint. The first difficulty is that this constraint is defined on the entire domain, leading for the discretized setting to a constraint for each cell. The problem lies again in the gradient calculation: for each constraint, a gradient should be calculated, which would lead to a number of gradient calculations equal to the amount of cells. Even with the adjoint approach, this becomes incredibly expensive because for each constraint, a new set of adjoint equations would have to be solved. 
To circumvent this issue, the state constraint could be reformulated equivalently as $\max _{\Omega} T \leq T_{\text {rec }}$, which is just a single constraint. However, the max-operator is non-differentiable, so it has to be replaced by an approximate differentiable functional. This approach is called constraint aggregation (see [14]). There are different choices for the aggregation functional of which the Kreisselmeier-Steinhauser (KS) functional, $h_{K S}$, is the most popular choice [15]. The constraint is now reformulated as follows:

$h_{K S}=\frac{1}{\rho} \log \frac{1}{\alpha} \int_{\Omega} \exp (\rho g) d \omega \leq 0$,

where $g=\frac{T}{T_{r e c}}$ and $\rho$ and $\alpha$ are parameters that can be tuned to let the functional approach the real $\max _{\Omega} T \leq T_{\text {rec }}$ constraint.

\section{Optimization of the tungsten volume fraction distribution in an ITER like monoblock}

In this section, the tungsten composition of a monoblock with ITER like geometry, taken from [16], is optimized and compared to the baseline ITER monoblock performance. To avoid designs which would be subject to severe erosion effects that are not modeled, a top layer of $3 \mathrm{~mm}$ thickness of pure tungsten is kept. Additionally, the cooling duct composition is held fixed as $\mathrm{CuCrZr}$. To aim for reduced temperatures, while trying to remain above the DBTT without imposing it as a hard constraint, $\tau$ is chosen to be 0.25 . The melting temperature will be computed using the linear mixture rule as well, although microscopically, the copper phase of the FGM might already melt at temperatures lower than the computed melting temperature. These issues will be discussed further in this section. The optimization is performed on a grid with 40000 cells and took approximately 3 hours to complete 100 optimization steps on a Dell Latitude E5570 with Intel i7 processor $(4 \times 2.6 \mathrm{GHz})$ and $16 \mathrm{~GB}$ DDR4 RAM.

Figure 1(a) shows the tungsten fraction in the optimized case. For reference: the tungsten fraction for the ITER case is given in figure 1(b). There is a clear gradient layer formed beneath the tungsten surface layers. This result can be explained as follows: to reach a uniform temperature to melt temperature ratio, the optimizer can either increase the tungsten fraction to obtain a higher melting temperature, yet lower thermal conduction coefficient, or decrease it for the opposite effect. At the top, it is impossible to lower the temperature significantly without lowering the temperature at the bottom too much. Therefore, the optimizer chooses to increase the tungsten fraction there locally. At the bottom, it decreases the tungsten fraction to obtain a lower melting temperature and hence reaching the desired ratio as good as possible.

On figure $1(\mathrm{c})$ and $1(\mathrm{~d})$, the temperature profile of the optimized and reference case is plotted, respectively. The surface temperature is reduced by roughly $200^{\circ} \mathrm{C}$ in comparison to the ITER design due to the reduction of the tungsten fraction beneath the top layer, hence proving that the optimized temperature profile is well below the recrystallization temperature and that introducing the FGM leads to a significantly lower overall temperature profile. At the bottom, the temperature retains values of the order of the DBTT of tungsten and lower, which might lead to unwanted local brittle behavior. A similar argument holds for the copper fraction: near the top layer, the temperature is around $800^{\circ} \mathrm{C}$, coming close to the melting temperature of copper. As stated before, this could cause the local microscopic copper volumes to melt. These issues could be avoided by including temperature constraints specifically for regions where copper or tungsten is present, which is left for future research.

Figure 1(e) and 1(f) give the temperature ratio for the optimized and reference design, respectively. Comparing both figures, it is clear that the optimized design outperforms the ITER design on this aspect. Therefore, it is concluded that the optimization works as expected and efficiently improves the design based on the given cost function.

To have a measure of the stresses in the monoblock, the Von Mises stress distribution, without considering yield stress, is calculated in a postprocessing step:

$$
\sigma_{V M}=\frac{\sqrt{\frac{1}{2}\left[\left(\sigma_{x x}-\sigma_{y y}\right)^{2}+\left(\sigma_{y y}-\sigma_{z z}\right)^{2}+\ldots\right.}}{\left.\left(\sigma_{z z}-\sigma_{x x}\right)^{2}+6 \sigma_{x y}^{2}+6 \sigma_{y z}^{2}+6 \sigma_{x z}^{2}\right]}
$$

The stresses $\sigma_{i j}$ are calculated by solving Navier's equation in 3D using a linear elastic and isotropic stress-strain relationship and assuming traction-free boundary conditions except for the bottom, which is assumed to be fixed everywhere. Figures $1(\mathrm{~g})$ and $1(\mathrm{~h})$ show the profiles of $\sigma_{V M}$ for the optimized design and ITER reference case at the plane $z=0$, respectively. The stresses at the original tungstencopper interface have been reduced, but this goes at the cost of introducing a new local stress concentration at the top layer material interface. This could be avoided by tuning $\tau$ to a desired value, or by introducing a new cost function based on the actual stress. Although the former is computationally less expensive and easier to evaluate, the latter will not require tuning and will target local stress concentrations directly and hence might better exploit the potential of FGMs. 

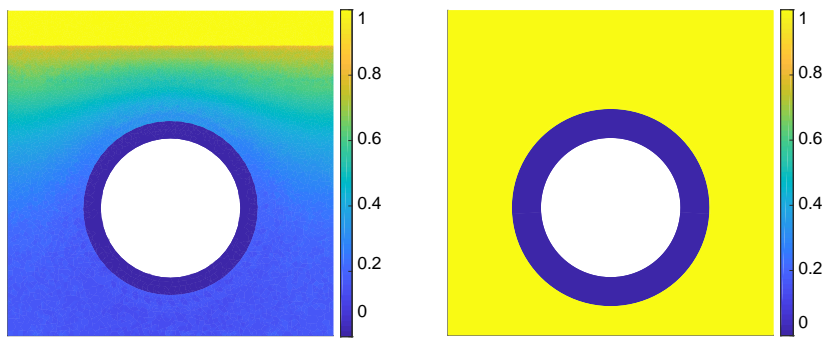

(a) Optimized divertor tungsten (b) ITER reference tungsten fraction distribution. fraction distribution.
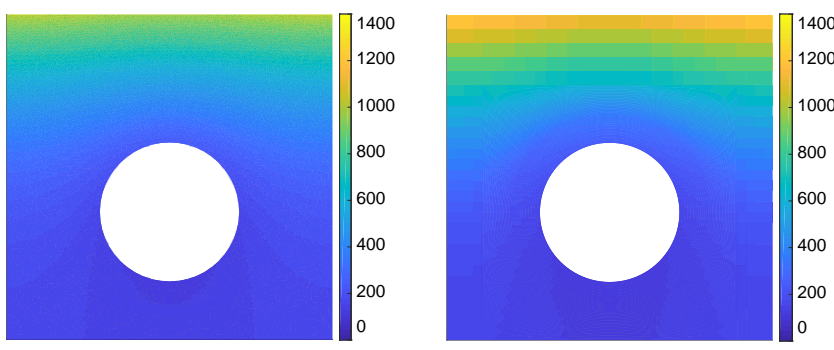

(c) Temperature distribution for (d) Temperature distribution optimized design $\left[{ }^{\circ} \mathrm{C}\right]$.
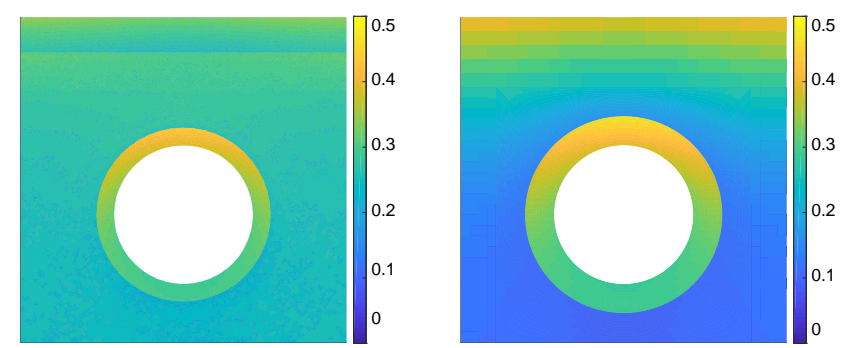

(e) $\frac{T}{T_{m}}$ for optimized design $\left[\frac{\mathrm{K}}{\mathrm{K}}\right]$.

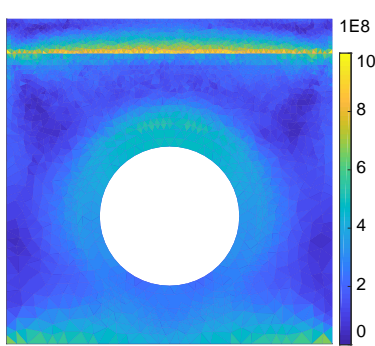

(f) $\frac{T}{T_{m}}$ for ITER design $\left[\frac{\mathrm{K}}{\mathrm{K}}\right]$.

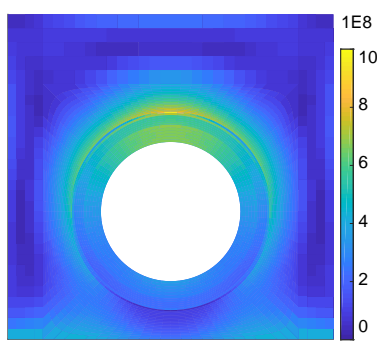

(g) $\sigma_{V M}$ for optimized design (h) $\sigma_{V M}$ for ITER design [Pa]. $[\mathrm{Pa}]$.

Figure 1. Composition, temperature distribution, temperature ratio and Von Mises stress measure for the optimized design (left column) and ITER reference case (right column)

\section{Conclusion}

A numerical optimization strategy has been developed to find the composition of a melt-injected W-Cu FGM that optimizes the fractional margin of the operation temperatures to the local melting temperatures while respecting the maximum temperature bound. The first results show clearly that the optimizer efficiently improves the design by reducing the given cost function. By imposing constraints on the temperature, it also achieves to keep the temperature profile beneath the recrystallization temperature.

However, the proposed cost function does not perform satisfactory as it can be insensitive to some local stress concentrations. Therefore, further research should focus on including a stress evaluation and penalization for the cost function. Ideally, this cost function is based on an appropriate failure criterion for the monoblock, which will most likely include some stress measure. Furthermore, additional constraints are needed to account in more detail for material limits. Future efforts should amongst others aim at accounting for the DBTT temperature of tungsten. Moreover, a maximum temperature for copper should be imposed to prevent melting of the copper volumes in the FGM. This can be done by extending the constraint aggregation methodology introduced in section 3 .

\section{Acknowledgements}

Sander Van den Kerkhof is a doctoral research fellow of the Research Foundation - Flanders (FWO) under grant number 37208. Maarten Blommaert is a postdoctoral research fellow of FWO and VITO, the Flemish Institute for Technological Research.

\section{References}

[1] T. Donné and W. Morris, "European Research Roadmap to the Realisation of Fusion Energy," tech. rep., EUROfusion, Sept. 2018.

[2] R. Pitts, S. Carpentier, F. Escourbiac, T. Hirai, V. Komarov, S. Lisgo, A. Kukushkin, A. Loarte, M. Merola, A. Sashala Naik, R. Mitteau, M. Sugihara, B. Bazylev, and P. Stangeby, "A full tungsten divertor for ITER: Physics issues and design status," Journal of Nuclear Materials, vol. 438, pp. S48-S56, July 2013.

[3] M. Li and J.-H. You, "Structural impact of armor monoblock dimensions on the failure behavior of ITERtype divertor target components: Size matters," Fusion Engineering and Design, vol. 113, pp. 162-170, Dec. 2016.

[4] M. Li, E. Werner, and J.-H. You, "Low cycle fatigue behavior of ITER-like divertor target under DEMOrelevant operation conditions," Fusion Engineering and Design, vol. 90, pp. 88-96, Jan. 2015.

[5] M. Li, E. Werner, and J.-H. You, "Fracture mechanical analysis of tungsten armor failure of a water-cooled divertor target," Fusion Engineering and Design, vol. 89, pp. 2716-2725, Nov. 2014.

[6] T. Hirai, F. Escourbiac, S. Carpentier-Chouchana, A. Fedosov, L. Ferrand, T. Jokinen, V. Komarov, A. Kukushkin, M. Merola, R. Mitteau, R. Pitts, W. Shu, M. Sugihara, B. Riccardi, S. Suzuki, and R. Villari, "ITER tungsten divertor design development and qualification program," Fusion Engineering and Design, vol. 88, pp. 1798-1801, Oct. 2013.

[7] Y. Itoh, M. Takahashi, and H. Takano, "Design of tungsten/copper graded composite for high heat flux 
components," Fusion Engineering and Design, vol. 31, pp. 279-289, 1996.

[8] G. Pintsuk, S. Brünings, J.-E. Döring, J. Linke, I. Smid, and $\mathrm{L}$. Xue, "Development of $\mathrm{W} / \mathrm{Cu}$ - functionally graded materials," Fusion Engineering and Design, vol. 66-68, pp. 237-240, Sept. 2003.

[9] M. Baelmans, M. Blommaert, W. Dekeyser, and T. Van Oevelen, "Achievements and challenges in automated parameter, shape and topology optimization for divertor design," Nuclear Fusion, vol. 57, Mar. 2017.

[10] W. Dekeyser, D. Reiter, and M. Baelmans, "Divertor Design through Shape Optimization," Contributions to Plasma Physics, vol. 52, pp. 544-549, June 2012.

[11] M. Blommaert, H. Heumann, M. Baelmans, N. R. Gauger, and D. Reiter, "Towards Automated Magnetic Divertor Design for Optimal Heat Exhaust," ESAIM: Proceedings and Surveys, vol. 53, pp. 49-63, Mar. 2016.

[12] M. Blommaert, W. Dekeyser, M. Baelmans, N. R. Gauger, and D. Reiter, "A practical globalization of one-shot optimization for optimal design of tokamak divertors," Journal of Computational Physics, vol. 328, pp. 399-412, Jan. 2017.

[13] A. Muller, D. Ewert, A. Galatanu, M. Milwich, R. Neu, J. Pastor, U. Siefken, E. Tejado, and J. You, "Melt infiltrated tungsten-copper composites as advanced heat sink materials for plasma facing components of future nuclear fusion devices," Fusion Engineering and Design, vol. 124 , pp. $455-459$, Nov. 2017.

[14] J. R. R. A. Martins and N. M. K. Poon, "On structural optimization using constraint aggregation," in 6th World Congress on Structural and Multidisciplinary Optimization, 2005.

[15] G. J. Kennedy and J. E. Hicken, "Improved constraintaggregation methods," Computer Methods in Applied Mechanics and Engineering, vol. 289, pp. 332-354, 2015.

[16] V. Komarov, "Thermal analysis of ITER Divertor PFU W-monoblocks with local shaping at surface heat flux," Tech. Rep. KRTXGK, ITER Office, Sept. 2013. 